$$
\text { Mary Ann Liebert, Inc. puldishers }
$$

\title{
Cannabidiol Does Not Dampen Responses to Emotional Stimuli in Healthy Adults
}

\author{
David L. Arndt and Harriet de Wit ${ }^{*}$
}

\begin{abstract}
Introduction: Cannabidiol (CBD) is a nonpsychoactive constituent of whole plant cannabis that has been reported to reduce anxiety-like behaviors in both pre-clinical and human laboratory studies. Yet, no controlled clinical studies have demonstrated its ability to reduce negative mood or dampen responses to negative emotional stimuli in humans. The objective of this study was to investigate the effects of CBD on responses to negative emotional stimuli, as a model for its potential anxiety-reducing effects.

Materials and Methods: The study used a double-blind, placebo (PLB)-controlled, within-subjects design in which 38 healthy, drug-free participants consumed oral CBD (300,600, and $900 \mathrm{mg}$ ) or PLB before completing several behavioral tasks selected to assess reactivity to negative stimuli. Dependent measures included emotional arousal to negative and positive visual stimuli, perceptual sensitivity to emotional facial expressions, attentional bias toward emotional facial expressions, and feelings of social rejection. In addition, subjective drug effects and physiological data were also gathered during each experimental session to assess drug effects.

Discussion: CBD did not dampen responses to negative emotional stimuli and did not affect feelings of social rejection. The high dose of CBD $(900 \mathrm{mg})$ marginally reduced attentional bias toward happy and sad facial expressions, and produced a slight increase in late-session heart rate. CBD did not produce detectable subjective effects or alterations in mood or anxiety.

Conclusion: These findings indicate that CBD has minimal behavioral and subjective effects in healthy volunteers, even when they are presented with emotional stimuli. Further research into the behavioral and neural mechanisms of CBD and other phytocannabinoids is needed to ascertain the clinical function of this drug.
\end{abstract}

Keywords: behavior; cannabidiol; emotional stimuli; psychopharmacology

\section{Introduction}

Cannabidiol (CBD), a constituent of cannabis, has received enormous public and scientific attention over the past decade. CBD citations in PubMed increased from 40 in 2000-2002 to 458 in 2014-2016. Although many of these refer to the potential of CBD to treat psychiatric or neurological disorders, ${ }^{1,2}$ there have been promising reports of its efficacy for treatment-resistant epilepsy and, combined with delta- 9 tetrahydrocannabinol (THC; Sativex ${ }^{\circledR}$ ), for multiple sclerosis. There is also evidence that single doses of CBD alter mood or behavior, either alone or in combination with other cannabinoids.

Several studies suggest that CBD has anxiolytic effects. Zuardi et al. ${ }^{3}$ reported that CBD (about $35 \mathrm{mg}$ ) reduced anxiety provoked by oral THC (about $70 \mathrm{mg}$ ) in normal volunteers. Later, Zuardi et al. ${ }^{4}$ reported that CBD (300 mg) reduced anxiety during a stressful public speaking task in healthy adults, to a similar extent as diazepam $(10 \mathrm{mg})$ and ipsaperone $(5 \mathrm{mg})$. In other studies, CBD reduced anxiety during public speaking in individuals with social anxiety disorder, ${ }^{5}$

Department of Psychiatry and Behavioral Neuroscience, University of Chicago, Chicago, Illinois.

*Address correspondence to: Harriet de Wit, PhD, Department of Psychiatry and Behavioral Neuroscience, University of Chicago, 5841 S. Maryland Avenue, MC 3077, Rm L485, Chicago, IL 60637, E-mail: hdew@uchicago.edu

(c) David L. Arndt et al., 2017; Published by Mary Ann Liebert, Inc. This is an Open Access article distributed under the terms of the Creative Commons Attribution License, which permits unrestricted use, distribution, and reproduction in any medium, provided the original work is properly cited. Mary Ann Liebert, Inc. offers reprint services for those who want to order professionally produced copies of articles published under the Creative Commons Attribution (CC BY) license. To obtain a price quote, email Reprints@liebertpub.com. Please include the article's title or DOI, quantity, and delivery destination in your email. 
reduced amygdala responses to fearful faces in healthy men, ${ }^{6,7}$ and reduced anxiety in response to a stressful imaging procedure in men. ${ }^{8} \mathrm{CBD}$ also produces anxiolytic-like effects in animal models. ${ }^{9}$ Together, these findings suggest that CBD may possess anxiolytic properties similar to those of known anxiolytic drugs.

The neural mechanisms by which CBD acts in the brain are poorly understood. CBD has low binding affinity for either $\mathrm{CB} 1$ or $\mathrm{CB} 2$ receptors. However, some in vivo studies indicate that the behavioral effects of CBD may be elicited indirectly through these receptors. ${ }^{10,11} \mathrm{CB} 1$ inverse agonists can block the behavioral effects of CBD in mouse models of fear conditioning, extinction, and marble burying behaviors. ${ }^{12,13} \mathrm{CBD}$ administered directly into key brain regions reduces anxiety-like behavior in rodents. ${ }^{14-16} \mathrm{CBD}$ may also reduce anxiety and alleviate other neurological disorders by enhancing anandamide through fatty acid amide hydrolase inhibition ${ }^{17,18}$ or by altering serotonergic (5-HT) neurotransmission, including actions as an indirect 5-HT1A agonist. ${ }^{1,18-21}$

In this study, we examined the effects of CBD on responses to negative emotional stimuli in healthy human volunteers. ${ }^{22}$ Single doses of anxiolytic and antidepressant drugs produce subtle changes in perception and responses to emotional stimuli in healthy individuals, effects that appear to predict their therapeutic efficacy. ${ }^{23-26}$ In this study, we used such measures to study the effects of $\mathrm{CBD}$. We tested participants' responses to images or words with negative affective content, reactivity to threatening emotional faces, and sensitivity to social rejection after oral $\operatorname{CBD}(0,300,600$, and $900 \mathrm{mg}$ ) in a double-blind design. We hypothesized that $\mathrm{CBD}$ would reduce reactivity to negative emotional stimuli.

\section{Materials and Methods \\ Design}

Healthy men and women aged 18-35 years participated in this four-session, within-subjects double-blind study. On each session, they received a single oral dose of CBD $(300,600$, and $900 \mathrm{mg}$ ) or placebo (PLB) in randomized order. The study was approved by the University of Chicago Biological Science Division Institutional Review Board, and procedures were in accordance with the Helsinki Declaration of 1975, as revised in 2008.

\section{Drug}

Oral CBD (300 mg/ml solution) or PLB vehicle was provided by Insys Therapeutics, Inc. (IND 125302). On each session, participants received a total of $4 \mathrm{ml}$ of fluid that included $1 \mathrm{ml}$ of sugar-free syrup to enhance palatability and to improve blinding (Ora-Sweet; Paddock Laboratories, Minneapolis, $\mathrm{MN}$ ), plus the appropriate volume of active $\mathrm{CBD}(0,1,2$ or $3 \mathrm{ml})$ and vehicle $(3,2,1$ or $0 \mathrm{ml})$. The solutions for each dose were matched on taste, physical characteristics, and volume. The drug was administered $2.5 \mathrm{~h}$ before subjects completed the behavioral tasks, based on evidence that CBD plasma levels are rising at this time. ${ }^{27,28}$

\section{Subjects}

Volunteers (19 men and 19 women) were recruited through posters, advertisements, and word-of-mouth referrals. Screening included a physical examination, an electrocardiogram, a psychiatric screening interview, and detailed drug use history. Individuals were excluded if they had cardiovascular problems, used prescription medications (except hormonal contraception in women), had a current DSM-V Axis $\mathrm{I}^{29}$ mood, anxiety, eating, or substance dependence disorder, or a lifetime history of a psychotic disorder. Women who were pregnant, nursing, or planning to become pregnant, anyone with less than a high-school education, not fluent in English, body mass index (BMI) less than 19 or more than 30 , or who reported using cannabis $>100$ times in their lifetime were also excluded to minimize possible tolerance to CBD.

\section{Study procedures}

Orientation. Participants who met criteria attended an orientation session to explain the study, obtain consent, and practice the study tasks. They agreed to fast after 11:00 am on the day of the sessions and abstain from alcohol and other drug use for $24 \mathrm{~h}$ before sessions. To mitigate expectancy effects, participants were told that they might receive a PLB, a stimulant, a sedative, or a cannabis-like drug (e.g., THC or CBD).

Study sessions. Sessions were conducted in a comfortable laboratory room from 1:00 pm to 5:45 pm, separated by at least 1 week. Upon arrival, subjects provided urine and breath samples to test for recent alcohol and drug use, and a pregnancy test (for women). Subjects who tested positive were rescheduled or dropped from the study. Participants then consumed a standardized snack (granola bar) and completed baseline (Time Point 1) measures of subjective mood, drug effects, and cardiovascular variables. These measures were obtained at regular 30-60 min intervals during 
the 4-h session. At 1:30 pm, participants ingested capsules containing CBD $(300,600$, and $900 \mathrm{mg})$ or PLB. For the next $2.5 \mathrm{~h}$ until the drug effect reached its expected peak, ${ }^{27,28}$ participants relaxed and watched a movie or read a book. From $4 \mathrm{pm}$ to $5 \mathrm{pm}$, they completed the tasks described hereunder to assess their reactivity to emotional stimuli. The tasks were presented in a counterbalanced order. Participants were discharged at 5:45 pm.

\section{Physiological measures}

Cardiovascular measures. Heart rate and blood pressure (BP) were measured using portable monitors (Omron Automatic Blood Pressure Monitor, Model No. BP791IT; Omron Healthcare, Inc., Lake Forest, IL). Mean arterial pressure (MAP; [systolic $\mathrm{BP}+2 \times$ diastolic $\mathrm{BP}] / 3$ ) was calculated.

\section{Subjective measures}

Profile of Mood States. The Profile of Mood States (POMS) is a validated measure of mood states consisting of 72 adjectives commonly used to describe momentary moods. ${ }^{30}$ The POMS is sensitive to the effects of moodaltering drugs in healthy volunteers. ${ }^{31,32}$

Drug Effects Questionnaire. The Drug Effects Questionnaire is a validated measure of subjective drug effects. $^{33,34}$ Participants indicate on a visual analog scale the extent they feel a drug effect, whether they like or dislike the drug effect, and whether given a choice would they want to take more of the drug.

\section{Behavioral tasks}

We based our predictions of CBD on previous studies investigating other pharmacological interventions in similar behavioral tasks. Specifically, drugs that are known to improve anxiety or depression (tryptophan supplementation, antidepressant medication, steroids, and benzodiazepines) tend to reduce or direct an individual's attention away from negative emotional stimuli. $^{26}$ Thus, we predicted that CBD would result in similar behavioral outcomes.

Emotional Stroop. The Emotional Stroop is a measure of emotional arousal adapted from the original Stroop color task. ${ }^{26,35,36}$ It consists of words with specific emotional connotations displayed in four different colors. It includes positive and negative words with religious, social, and emotional content. For example, religiouspositive words included "angel," "paradise," and "divine," and social-negative words included "alone," "unwanted," and "disliked." It also includes a "color control" in which names of colors were presented in different colored font. ${ }^{36}$ Participants indicate the color of each word's text by pressing a corresponding key on a keyboard. Longer reaction times are expected for mismatched color and text, and longer reaction times with emotional words are indicative of a greater emotional response (longer fixation) to emotional words. ${ }^{37,38}$ Previous studies have shown that changes in tryptophan depletion can increase interference of words with negative connotations. ${ }^{39-41}$ Interestingly, CBD has also been shown to suppress tryptophan depletion in ways that suggest a neural mechanism by which antidepressant or anxiolytic effects of cannabinoids might be linked to serotonergic neurotransmission. ${ }^{42}$ Thus, it was predicted that CBD would blunt the emotional response toward words with negative connotations, resulting in faster reaction times toward negative words.

International Affective Picture System. The International Affective Picture System (IAPS) was used as a measure of emotional arousal ${ }^{43}$ to visual stimuli with affective content. Participants viewed standardized positive, negative, and neutral pictures from the IAPS. The negative and positive images were matched on degree of valence and arousal. Positive, negative, and neutral images were also categorized as "social" or "nonsocial" by the experimenters. Subjects rated both the positivity and the negativity of each image. It was predicted that $\mathrm{CBD}$ would reduce negativity ratings to negative pictures.

Dynamic Emotion Identification Task. The Dynamic Emotion Identification Task (DEIT) is a measure of sensitivity to detecting facial expressions, created for use in our laboratory. ${ }^{44}$ Participants viewed dynamically developing facial expressions comprising $2 \%$ morphs from a neutral face to a $100 \%$ expression of an emotion, happy, sad, angry, and fearful. Participants responded as soon as they could correctly identify the emotion expressed. Emotion identification was quantified as the intensity $(0-100 \%)$ of the face when the participant responded on trials when they correctly identified the emotion. Based on previous studies investigating pharmacologically induced reductions in reactivity to negative emotional stimli, ${ }^{25,26}$ we predicted that CBD would reduce sensitivity to negative facial expressions (i.e., a higher intensity of expression needed to identify sadness, anger, and fear). 
Attentional Bias Task. The Attentional Bias Task (ABT) measures attention toward emotional stimuli (facial expressions) and was adapted from Garner et al. ${ }^{45}$ Participants viewed a neutral face and one with an emotional expression ${ }^{46}$ side-by-side. After $500 \mathrm{msec}$ viewing, a white dot appeared in place of one of the faces and participants were instructed to indicate its location. Trials were separated by $750-1,250 \mathrm{msec}$. Attentional bias toward emotional facial expressions was indicated by a longer shorter reaction time for the emotional expression. ${ }^{47}$ Based on previous studies in nonclinical participants investigating the effects of serotonergic antidepressants and anxiolytics on emotional visual probe tasks, ${ }^{26}$ we predicted that $\mathrm{CBD}$ would reduce attentional bias (i.e., lengthen reaction times) to negative facial expressions (anger, sadness, and fear).

Cyberball. This is a measure of social acceptance and ostracism. ${ }^{48-50}$ Participants played two virtual games of toss with two other "players" who were represented as animated icons on the computer screen. Participants were told that they represented one of the three players. During the first game (acceptance), the ball was tossed equally among the three players. During the second game (rejection), the participant was excluded. After each game, participants rated their levels of mood and self-esteem. We have previously found that MDMA, a drug that has prosocial and mood-enhancing effects, can blunt the effects of simulated social rejection. ${ }^{51}$ It was predicted here that CBD would blunt the rejectioninduced reduction in mood and self-esteem. Participants also estimated the percentage of throws they received during each game.

\section{Data analyses}

The physiological and subjective effects of CBD were analyzed by two-way (drug $\times$ time) repeated measures analysis of variance (ANOVA). Behavioral tasks (Emotional Stroop, IAPS, DEIT, ABT, and Cyberball) were analyzed through a series of two-way mixed factorial ANOVAs. Significant effects were followed by post hoc comparisons of the estimated marginal means. Bonferroni corrections for multiple comparisons within each dependent measure were utilized to maintain a family-wise error rate of 0.05 .

\section{Results}

Participants

One person tested positive for cannabis and was dropped from the study. Table 1 illustrates that most of the
Table 1. Characteristics of the 38 Participants Who Completed the Cannabidiol Study

\begin{tabular}{lc}
\hline Gender (M:F) & $19: 19$ \\
Age (years) & $23.6(0.66)$ \\
Education (years) & $15.2(0.27)$ \\
Race & \\
Caucasian & 22 \\
African American & 10 \\
Other & 6 \\
Recent (past month) substance use & \\
Alcohol (drinks/week) & $5.28(0.64) ; n=35$ \\
Cigarettes (cigs/week) & $8.58(5.32) ; n=7$ \\
Caffeine (cups/day) & $1.32(0.23) ; n=33$ \\
Cannabis (times/month) & $5.12(1.02) ; n=14$ \\
Lifetime substance use (\% ever used) & \\
Cannabis & 94.7 \\
Hallucinogens & 23.7 \\
Stimulants & 15.8 \\
Opiates & 7.9 \\
MDMA & 18.4 \\
Sedatives & 5.3 \\
\hline
\end{tabular}

Age, education, and past month recent substance use are listed as mean (SEM). For recent substance use, the mean and SEM were calculated using only subjects who reported any recent use of the drug ( $n$ for each drug type is shown). Remaining subjects reported no recent use of the drugs. Race is number of individuals who identify as such ("Other" is mainly Asian). Lifetime substance use refers to nonmedical use only.

SEM, standard error of the mean.

remaining 38 subjects were in their early 20 s and reported light-to-moderate recent use of alcohol, nicotine, caffeine, and cannabis.

\section{Physiological response}

CBD had no effect on MAP ( $p>0.05$ ). CBD (300 and $600 \mathrm{mg}$ ) did not affect heart rate, and only the highest dose of CBD $(900 \mathrm{mg})$ resulted in a slight increase in heart rate at the last time point of the session, indicated by a significant drug $\times$ time interaction effect, $F(15$, 540) $=1.77, p=0.036$.

Subjective drug effects

CBD did not alter ratings of mood states (POMS) and subjects did not report feeling a drug effect, or liking or disliking it (all p's >0.05).

\section{Emotional Stroop}

As expected, subjects took longer to identify the name of the colored words when the text of the word was in a different color, $F(2,70)=85.08, p<0.001$. However, they did not exhibit longer reaction times with negative emotional words, and CBD had no effect on reaction times for any words (Fig. 1; all $p$ 's $>0.05$ ).

International Affective Picture System

As expected, subjects rated positive images more positively (i.e., higher positivity ratings, $F(2,72)=182.89$, 


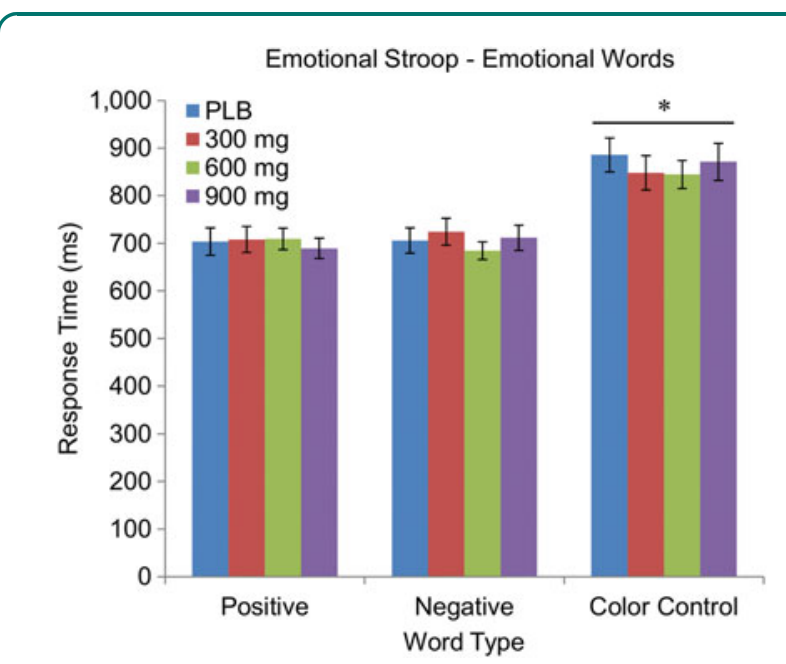

FIG. 1. Mean \pm SEM reaction times for emotional words in the Emotional Stroop task. Asterisk $\left(^{*}\right)$ indicates that both the reaction times for the negative and positive words were significantly shorter than the reaction times for the color control words, but the reaction times for the negative and positive words did not significantly differ from one another. CBD had no effect on reaction times for any words. CBD, cannabidiol; SEM, standard error of the mean.

$p<0.001)$ and negative images more negatively [i.e., higher negativity ratings, $F(2,72)=393.24, p<0.001]$. However, CBD did not alter ratings of either positive or negative stimuli (all $p$ 's $>0.05$; Fig. 2).

\section{Dynamic Emotion Identification Task}

As expected, subjects identified angry faces significantly earlier than any of the other facial emotions, $F(3,108)=18.30, p<0.001$. CBD did not affect the ability to identify any expressions, regardless of the emotion (Fig. 3; all p's > 0.05).

\section{Attentional Bias Task}

With all emotions taken together, CBD affected attentional bias [drug, $F(3,102)=3.07, p=0.031$ ], but this effect was no longer significant after correcting for multiple comparisons (all $p$ 's $>0.05$ ). CBD did not affect attentional bias toward specific facial expressions, either negative (anger, sadness, and fear) or happy (Fig. 4). The absence of an interaction between drug and emotion $(p>0.05)$ indicates that the effect of $\mathrm{CBD}$ on attentional bias was not significantly specific to certain emotions.

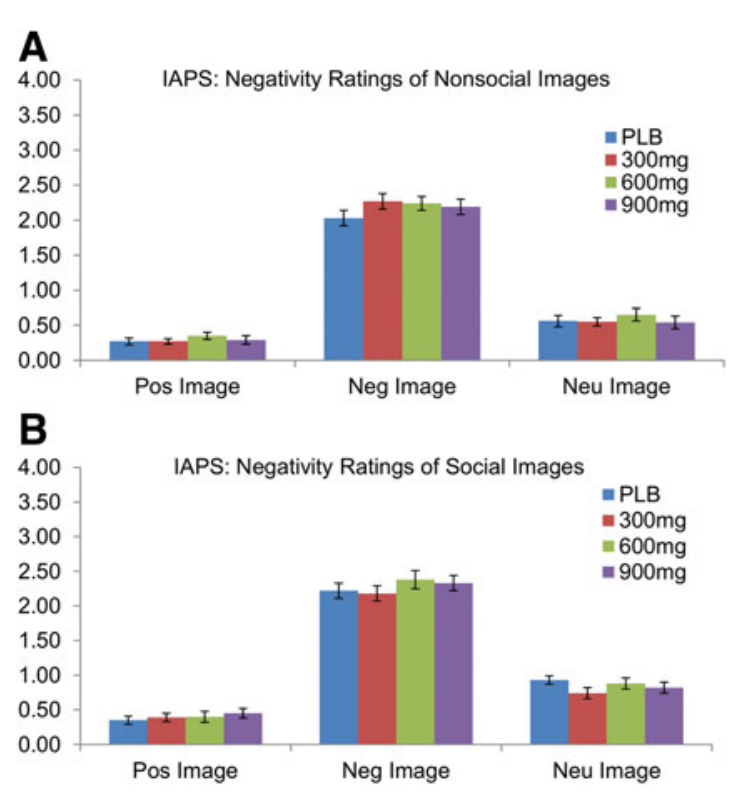

FIG. 2. Mean \pm SEM negativity ratings for (A) nonsocial and (B) social images of positive, negative, or neutral valence in the IAPS picture rating task. All image valence groups significantly differed from all other image valence groups, but CBD did not alter ratings of either positive or negative stimuli. A similar trend in positivity ratings was observed for both nonsocial and social images. IAPS, International Affective Picture System.

\section{Cyberball}

Subjects reported more negative mood, $F(1,34)=127.26$, $p<0.001$ and lower self-esteem, $F(1,34)=112.54$, $p<0.001$ during the "rejection" compared with the "acceptance" phase of the task, and correctly noted that they received fewer throws, $F(1,34)=440.26, p<0.001$ (data not shown). However, CBD did not change their responses during either phase (Fig. 5; all $p$ 's $>0.05$ ).

\section{Discussion}

Single doses of CBD (300, 600, and $900 \mathrm{mg}$ oral) did not dampen responses to negative emotional stimuli in healthy volunteers. The highest dose of CBD (900 mg) produced a slight, delayed increase in heart rate 240 min after administration (Fig. 6), and decreased attentional bias toward emotional facial expressions. But, CBD produced no detectable subjective effects (e.g., "feeling" a drug effect or ratings of momentary mood states) and had little effect on reactions to negative emotional stimuli on standardized tasks. The drug was well 


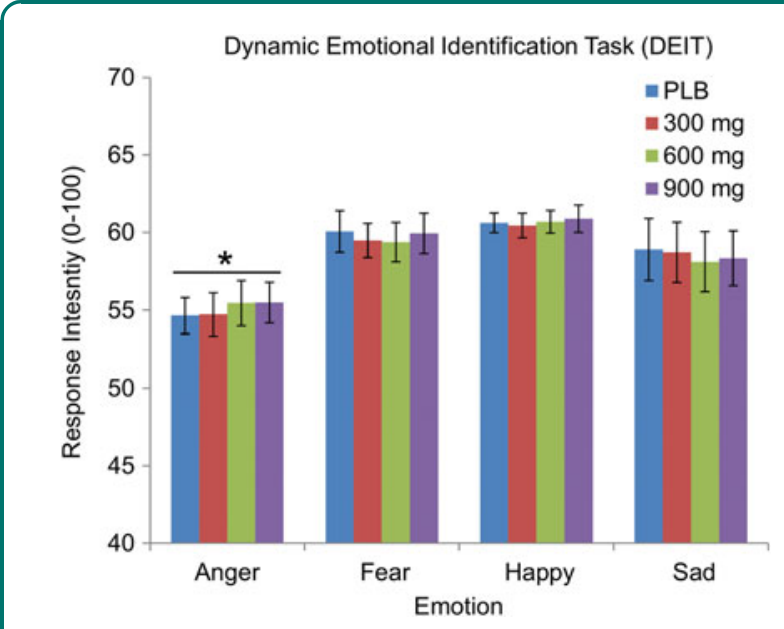

FIG. 3. Mean $\pm S E M$ response intensity of facial expressions at time of identification in the DEIT. Asterisk $\left(^{*}\right)$ indicates that participants identified angry faces significantly faster than any of the other facial emotions. None of the other facial emotions differed from one another, and CBD did not affect the ability to identify facial expressions, regardless of emotion. DEIT, Dynamic Emotion Identification Task.

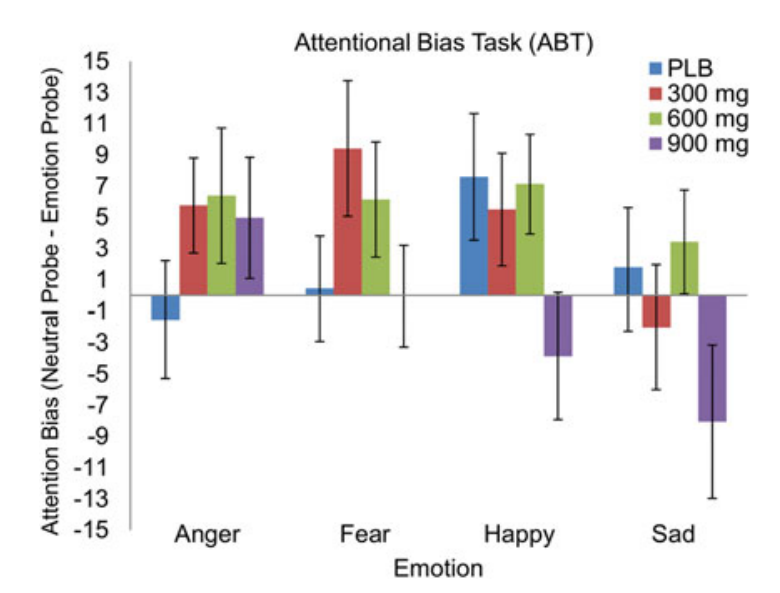

FIG. 4. Mean $\pm S E M$ attentional bias scores toward angry, fearful, happy, or sad facial expressions. With all emotions taken together, CBD affected attentional bias $(p=0.031)$. This effect of CBD was not specific to certain emotions.

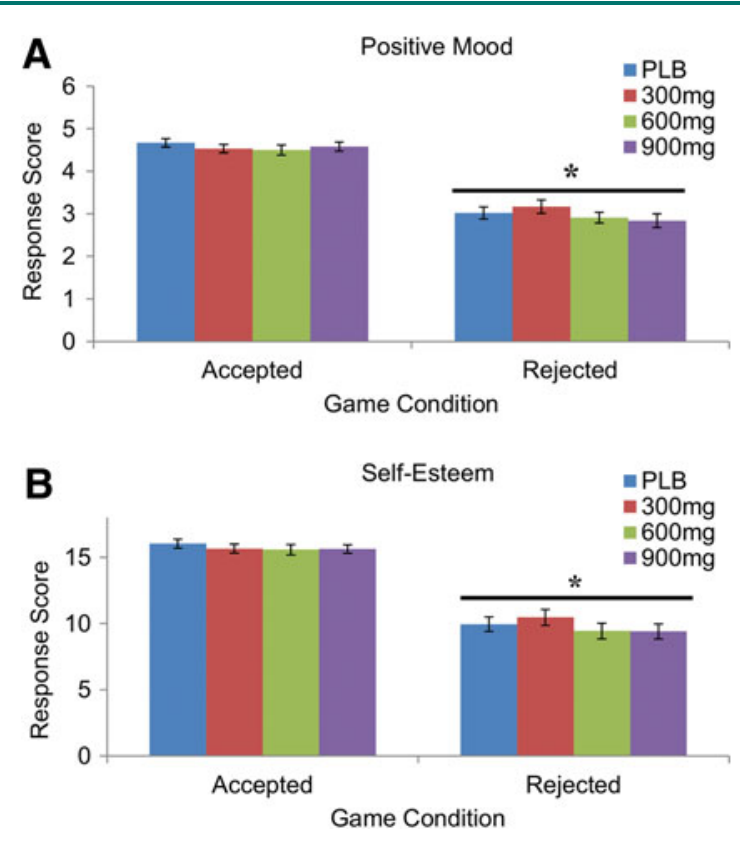

FIG. 5. Mean \pm SEM feelings of positive mood (A) and self-esteem (B) after the Cyberball games conditions of "accepted" and "rejected." Asterisks (*) indicate that subjects reported reduced feelings of positive mood and self-esteem after the rejection game, but CBD did not change their responses.

tolerated in these healthy young adults, and no participants experienced any adverse effects.

Previous findings on the effects of $\mathrm{CBD}$ on mood or subjective state have been mixed. Several previous studies have suggested that CBD has stress-dampening effects, either alone or when it is administered in combination with other constituents of the cannabis plant. ${ }^{38}$ Our finding that CBD had little or no effect on behavioral measures of negative emotionality is apparently inconsistent with the prior reports of anxiolytic effects. However, our findings are consistent with other recent reports that CBD has minimal psychological or behavioral effects. In particular, Babalonis et al. ${ }^{52}$ reported that oral CBD $(200,400$, and $800 \mathrm{mg})$ produced no detectable subjective or behavioral effects in regular cannabis users on measures of psychomotor performance and selective attention. In a related report, Haney et al. ${ }^{53}$ reported that $\mathrm{CBD}$ also did not change responses to cannabis.

An important shortcoming of this study was the absence of pharmacokinetic data to confirm that the drug 

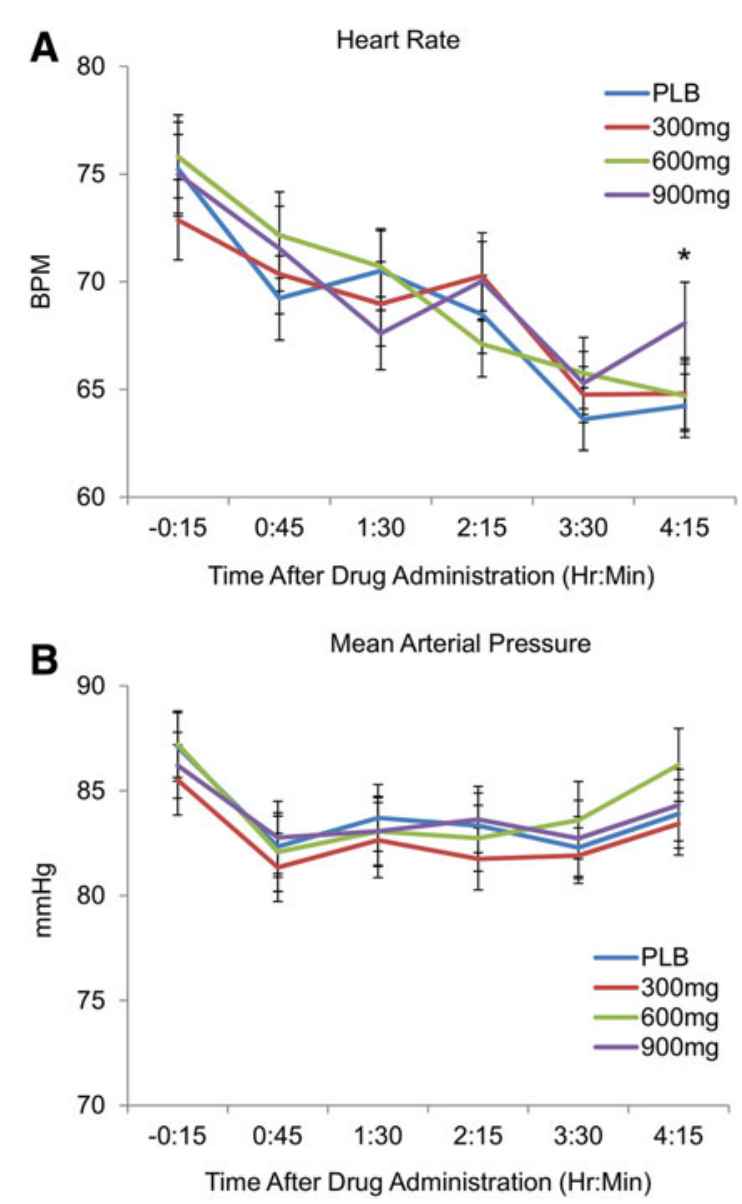

FIG. 6. Mean $\pm \operatorname{SEM}(\mathbf{A})$ heart rate and (B) blood pressure (BP) throughout the experimental session before and after CBD $(300,600$, and $900 \mathrm{mg}$ ) or placebo administration. Asterisk (*) indicates that the highest dose of CBD (900 mg) resulted in a slight increase in heart rate at the last time point of the session. CBD had no effect on BP (MAP, $p>0.05$ ). MAP, mean arterial pressure.

was absorbed and that the participants were exposed to the intended plasma concentrations of drug. Because the drug, unexpectedly, had little effect on any outcome measure, we cannot be assured that the appropriate dose was delivered. Furthermore, Haney et al. ${ }^{53}$ reported a great deal of inter-individual variability in the peak plasma levels of CBD: among the eight participants in their study who received $800 \mathrm{mg}$ CBD, peak plasma levels ranged from 1.6 to $271.9 \mathrm{ng} / \mathrm{ml}$ for the 6-h session, and the time to peak ranged from 120 to $360 \mathrm{~min}$. This level of variability makes it difficult to measure the effects of the drug, and, if present in our study, could have contributed to our negative findings. Unpublished data from the supplier of the CBD formulation used here indicated that peak plasma CBD concentrations after a single dose occurred $4.5 \mathrm{~h}$ after oral administration (standard deviation [SD] 1.62 in 20 healthy volunteers), suggesting that absorption here was adequate and plasma levels were rising when we implemented our behavioral tasks. On a separate issue, it also would be of interest to study the effects of chronic doses of CBD to determine whether steadystate concentrations of CBD produce detectable behavioral effects.

Little is known about effects of CBD on attentional bias. One study ${ }^{54}$ compared attentional bias to drug and food stimuli in individuals who reported smoking high CBD:THC strains compared with low CBD:THC ratios, and found that high CBD:THC smokers showed lower attentional bias to drug and food stimuli. However, it is difficult to relate those findings to the present results because of differences in drugs, participants, and study stimuli. It remains to be determined whether CBD truly alters attentional bias to emotional stimuli.

One question to be addressed in any study, especially one with "negative" findings, is whether the measures used were sensitive to the purported drug effect. In this case, we used four tasks assessing reactivity to emotional stimuli, and each task was previously shown to be sensitive to the effects of psychoactive drugs. Moreover, our tasks provided orderly and expected effects, independently of CBD administration. On the DEIT, subjects were quicker to identify angry facial expressions than other emotions, as previously reported, ${ }^{44}$ and other drugs are known to alter DEIT performance. ${ }^{44,55}$ Yet, we found no effect with CBD. On the IAPS task, participants rated positive pictures positively and negative pictures negatively, and previous studies have shown that stimulant drugs alter ratings of images. ${ }^{44,55}$ Again, CBD did not affect responses to images of any valence. In the Cyberball task, participants reported greater feelings of social rejection after the exclusion game in this study, and we have previously reported that certain psychoactive drugs reduce feelings of rejection. ${ }^{51}$ Yet, CBD did not blunt feelings of rejection in this study. Finally, on the ABT, the mean reaction times in this study were comparable to those in previous studies, ${ }^{47}$ and on the Stroop task, we detected the usual slowing on the color control condition, but CBD did not alter performance in either of these measures. Thus, CBD had little or no effect on tasks with known sensitivity. 
This study had limitations. We assessed attentional bias using reaction time to detect a stimulus, whereas previous studies used eye gaze, measured with electrooculography $^{44}$ (EOG). It is possible that the EOG measure provides a more sensitive index of attention. It is possible that the emotional stimuli used here were not salient enough to induce negative mood states or anxiety, or that the mood effects of CBD are only evident in individuals with high levels of anxiety, because of an underlying trait, or because of a contextual stressor (an anxiogenic drug or a stressful task ${ }^{4,5}$ ), as in prior reports of anxiolysis with CBD. Thus, it is possible that we did not detect a mood effect because participants were not experiencing anxiety at the time of CBD administration.

\section{Conclusions}

This study suggests that oral CBD does not alter responses to emotional stimuli, or produce anxiolyticlike effects in healthy human subjects. The absence of effect of CBD is consistent with another recent, carefully controlled laboratory study. ${ }^{52,53}$ Cannabinoids as a class of drugs are understudied, despite their widespread use for both recreational and therapeutic reasons. Yet, little is known about the active constituents and their effects on mood and behavior. Further research is needed to assess the therapeutic potential of cannabis constituents such as CBD. Studies of chronic, as well as acute administration of these drugs, as well as studies in at-risk individuals, are needed. Studies of the pharmacokinetic characteristics and the mechanisms of action are also essential. This research is critical to establish the brain actions, behavioral effects, and potential toxicity of this widely used class of drugs.

\section{Acknowledgments}

This study was supported by Insys Therapeutics, Inc., and by DA02812. Dr. Arndt was supported by T32GM007019. The authors thank Joel Cavallo for early contributions to this work.

\section{Author Disclosure Statement}

No competing financial interests exist.

\section{References}

1. Campos AC, Fogaça MV, Sonego AB, et al. Cannabidiol, neuroprotection and neuropsychiatric disorders. Pharmacol Res. 2016;112:119-127.

2. Zhornitsky S, Potvin S. Cannabidiol in humans - the quest for therapeutic targets. Pharmaceuticals. 2012;5:529-552.

3. Zuardi AW, Shirakawa I, Finkelfarb E, et al. Action of cannabidiol on the anxiety and other effects produced by $\Delta^{9}$-THC in normal subjects. Psychopharmacology (Berl). 1982;76:245-250.
4. Zuardi AW, Cosme RA, Graeff FG, et al. Effects of ipsapirone and cannabidiol on human experimental anxiety. J Psychopharmacol. 1993;7:82-88.

5. Bergamaschi MM, Queiroz RHC, Chagas MHN, et al. Cannabidiol reduces the anxiety induced by simulated public speaking in treatment-naive social phobia patients. Neuropsychopharmacology. 2011;36:1219-1226.

6. Bhattacharyya S, Morrison PD, Fusar-Poli P, et al. Opposite effects of [Delta]-9-tetrahydrocannabinol and cannabidiol on human brain function and psychopathology. Neuropsychopharmacology. 2009;35:764-774.

7. Fusar-Poli P, Crippa JA, Bhattacharyya S, et al. Distinct effects of delta 9-tetrahydrocannabinol and cannabidiol on neural activation during emotional processing. Arch Gen Psychiatry. 2009;66:95-105.

8. Crippa JAdS, Zuardi AW, Garrido GEJ, et al. Effects of cannabidiol (CBD) on regional cerebral blood flow. Neuropsychopharmacology. 2003;29: 417-426.

9. Schier ARM, Ribeiro NPO, Hallak JEC, et al. Cannabidiol, a Cannabis sativa constituent, as an anxiolytic drug. Rev Bras Psiquiatr. 2012;34:104-110.

10. Thomas A, Ross RA, Saha B, et al. 6"-Azidohex-2"-yne-cannabidiol: a potential neutral, competitive cannabinoid CB 1 receptor antagonist. Eur J Pharmacol. 2004;487:213-221

11. Thomas A, Baillie GL, Phillips AM, et al. Cannabidiol displays unexpectedly high potency as an antagonist of $C B 1$ and $C B 2$ receptor agonists in vitro. Br J Pharmacol. 2007;150:613-623.

12. Casarotto PC, Gomes FV, Resstel LB, et al. Cannabidiol inhibitory effect on marble-burying behaviour: involvement of $\mathrm{CB} 1$ receptors. Behav Pharmacol. 2010;21:353-358.

13. Stern CA, Gazarini L, Takahashi RN, et al. On disruption of fear memory by reconsolidation blockade: evidence from cannabidiol treatment. Neuropsychopharmacology. 2012;37:2132-2142.

14. Gomes FV, Resstel LB, Guimarães FS. The anxiolytic-like effects of cannabidiol injected into the bed nucleus of the stria terminalis are mediated by 5-HT1A receptors. Psychopharmacology (Berl). 2011;213:465-473.

15. Fogaca MV, Reis FMCV, Campos AC, et al. Effects of intra-prelimbic prefrontal cortex injection of cannabidiol on anxiety-like behavior: involvement of $5 \mathrm{HT} 1 \mathrm{~A}$ receptors and previous stressful experience. Eur Neuropsychopharmacol. 2014;24:410-419.

16. Hsiao YT, Yi PL, Li CL, et al. Effect of cannabidiol on sleep disruption in duced by the repeated combination tests consisting of open field and elecated plus-maze in rats. Neuropharmacology. 2012;62:373-384.

17. Deutsch DG. A personal retrospective: elevating anandamide (AEA) by targeting fatty acid amide hydrolase (FAAH) and the fatty acid binding proteins (FABPs). Front Pharmacol. 2016;7:370.

18. Hurd YL. Cannabidiol: swinging the marijuana pendulum from "weed" to medication to treat the opioid epidemic. Trends Neurosci. 2017;40:124-127.

19. Russo EB, Burnett A, Hall B, et al. Agonistic properties of cannabidiol at 5HT1a receptors. Neurochem Res. 2005;30:1037-1043.

20. Scuderi C, Filippis DD, luvone $T$, et al. Cannabidiol in medicine: a review of its therapeutic potential in CNS disorders. Phytother Res. 2009;23: 597-602.

21. Campos, AC, Guimarães, FS. Involvement of $5 \mathrm{HT} 1 \mathrm{~A}$ receptors in the anxiolytic-like effects of cannabidiol injected into the dorsolateral periaqueductal gray of rats. Psychopharmacology (Berl). 2008;199:223.

22. Pringle A, Browning $M$, Cowen PJ, et al. A cognitive neuropsychological model of antidepressant drug action. Prog Neuropsychopharmacol Biol Psychiatry. 2011;35:1586-1592.

23. Harmer CJ, Heinzen J, O'Sullivan U, et al. Dissociable effects of acute antidepressant drug administration on subjective and emotional processing measures in healthy volunteers. Psychopharmacology (Berl). 2008;199:495-502.

24. Harmer CJ, Bhagwagar Z, Perrett DI, et al. Acute SSRI administration affects the processing of social cues in healthy volunteers. Neuropsychopharmacology. 2003;28:148-152.

25. Harmer CJ. Serotonin and emotional processing: does it help explain antidepressant drug action? Neuropharmacology. 2008;55:1023-1028.

26. Browning $M$, Holmes EA, Harmer CJ. The modification of attentional bias to emotional information: a review of the techniques, mechanisms, and relevance to emotional disorders. Cogn Affect Behav Neurosci. 2010;10:8-20.

27. Grotenhermen F. Cannabinoids for therapeutic use. Am J Drug Deliv. 2004;2:229-240.

28. Agurell S, Carlsson S, Lindgren JE, et al. Interactions of $\Delta 11$ tetrahydrocannabinol with cannabinol and cannabidiol following oral 
administration in man. Assay of cannabinol and cannabidiol by mass fragmentography with cannabinol and cannabidiol following oral administration in man. Assay of cannabinol and cannabidiol by mass fragmentography. Experientia. 1981;37:1090-1092.

29. American Psychiatric Association. Diagnostic and statistical manual of mental disorders (DSM- ${ }^{\circledR}$ ). American Psychiatric Pub.: Arlington, VA, 2013.

30. McNair D, Lorr M, Droppleman L. Profile of mood states. Educational and Industrial Testing Service: San Diego, CA, 1981, pp. 1-29.

31. de Wit $H$, Griffiths RR. Testing the abuse liability of anxiolytic and hypnotic drugs in humans. Drug Alcohol Depend. 1991;28:83-111.

32. Johanson $\mathrm{CE}$, Uhlenhuth EH. Drug preference and mood in humans: diazepam. Psychopharmacology (Berl). 1980;71:269-273.

33. Fischman MW, Foltin RW. Utility of subjective-effects measurements in assessing abuse liability of drugs in humans. Br J Addict. 1991;86:1563-1570.

34. Morean $\mathrm{ME}$, de Wit $\mathrm{H}$, King $\mathrm{AC}$, et al. The drug effects questionnaire: psychometric support across three drug types. Psychopharmacology (Berl). 2013;227:177-192

35. Williams JMG, Mathews A, MacLeod C. The Emotional Stroop task and psychopathology. Psychol Bull. 1996;120:3.

36. Stroop JR. Studies of interference in serial verbal reactions. J Exp Psychol. 1935; 18:643.

37. Dresler T, Mériau K, Heekeren HR, et al. Emotional Stroop task: effect of word arousal and subject anxiety on emotional interference. Psychol Res. 2009;73:364-371.

38. Gotlib IH, McCann CD. Construct accessibility and depression: an examination of cognitive and affective factors. J Pers Soc Psychol. 1984;47:427.

39. Evers EAT, Van der Veen FM, Jolles J, et al. Acute tryptophan depletion improves performance and modulates the BOLD response during a Stroop task in healthy females. Neuroimage. 2006;32:248-255.

40. Hayward G, Goodwin GM, Cowen PJ, et al. Low-dose tryptophan depletion in recovered depressed patients induces changes in cognitive processing without depressive symptoms. Biol Psychiatry. 2005;57:517-524.

41. Munafò MR, Hayward G, Harmer C. Selective processing of social threat cues following acute tryptophan depletion. J Psychopharmacol. 2006;20:33-39.

42. Jenny $M$, Schröcksnadel $S$, Überall $F$, et al. The potential role of cannabinoids in modulating serotonergic signaling by their influence on tryptophan metabolism. Pharmaceuticals. 2010;3:2647-2660.

43. Lang PJ, Bradley MM, Cuthbert BN. International affective picture system (IAPS): technical manual and affective ratings. NIMH Center for the study of emotion and attention. University of Florida: Birmingham, AL, 1999.

44. Wardle M, Garner M, Munafò $M$, et al. Amphetamine as a social drug: effects of $d$-amphetamine on social processing and behavior. Psychopharmacology (Berl). 2012;223:199-210.

45. Garner M, Mogg K, Bradley BP. Orienting and maintenance of gaze to facial expressions in social anxiety. J Abnorm Psychol. 2006;115:760-770.

46. Goeleven E, De Raedt R, Leyman L, et al. The Karolinska Directed Emotional Faces: a validation study. Cogn Emot. 2008;22:1094-1118.
47. Clark-Elford R, Nathan PJ, Auyeung B, et al. Effects of oxytocin on attention to emotional faces in healthy volunteers and highly socially anxious males. Int J Neuropsychopharmacol. 2015;18:pyu012.

48. Zadro L, Williams KD, Richardson R. How low can you go? Ostracism by a computer is sufficient to lower self-reported levels of belonging, control, self-esteem, and meaningful existence. J Exp Soc Psychol. 2004;40: 560-567.

49. Williams KD, Jarvis B. Cyberball: a program for use in research on interpersonal ostracism and acceptance. Behav Res Methods. 2006;38: $174-180$

50. Liu JC, Mulick D, Chee MW. Odd one out: social ostracism affects selfreported needs in both sleep-deprived and well-rested persons. J Sleep Res. 2014;23:448-457.

51. Frye CG, Wardle MC, Norman GJ, et al. MDMA decreases the effects of simulated social rejection. Pharmacol Biochem Behav. 2014;117:1-6.

52. Babalonis S, Haney M, Malcolm RJ, et al. Oral cannabidiol does not produce a signal for abuse liability in frequent marijuana smokers. Drug Alcohol Depend. 2017;172:9-13.

53. Haney M, Malcolm RJ, Babalonis S, et al. Oral cannabidiol does not alter the subjective, reinforcing or cardiovascular effects of smoked cannabis Neuropsychopharmacology. 2016;41:1974-1982.

54. Morgan CJ, Freeman TP, Schafer GL et al. Cannabidiol attenuates the appetitive effects of $\Delta 9$-tetrahydrocannabinol in humans smoking their chosen cannabis. Neuropsychopharmacology. 2010;35:1879-1885.

55. Wardle $\mathrm{M}$, de Wit $\mathrm{H}$. Effects of amphetamine on reactivity to emotional stimuli. Psychopharmacology (Berl). 2012;220:143-153.

Cite this article as: Arndt DL, de Wit $\mathrm{H}$ (2017) Cannabidiol does not dampen responses to emotional stimuli in healthy adults, Cannabis and Cannabinoid Research 2:1, 105-113, DOI: 10.1089/can.2017.0014.
Publish in Cannabis and Cannabinoid Research

Cannabis and

Cannabinoid

Research
- Immediate, unrestricted online access

- Rigorous peer review

- Compliance with open access mandates

- Authors retain copyright

- Highly indexed

- Targeted email marketing 